\title{
Optimal inspection policy for three-state systems monitored by control charts
}

\author{
Shaomin $\mathrm{Wu}^{1}$ \\ School of Applied Sciences, Cranfield University, Bedfordshire MK43 0AL, United \\ Kingdom \\ Wenbin Wang \\ Salford Business School, University of Salford, Salford M45 4WT, UK. E City \\ University of Hong Kong, Hong Kong
}

\begin{abstract}
This paper presents the formulations of the expected long-run cost per time unit for a system monitored by a static control chart and by an adaptive control chart respectively. The static chart has a fixed sampling interval and a fixed sample size. The adaptive chart has a fixed sample size but variable sampling intervals. The system is supposed to have three states, normal working state, failure delay time state, and failed state. Two levels of repair are used to maintain the system. A minor repair is used to restore the system if a detectable defect is confirmed by an inspection. A major repair will be performed if the system fails. The expected cost per time unit for maintaining such a system is obtained. The objective of such analysis is to find an optimal sampling policy for the inspection process. An artificially generated data example and a real data example are used to compare the expected cost per time unit for both the static and adaptive control charts.
\end{abstract}

Keywords: Maintenance policy; control chart; condition-based maintenance; multi-state system; inspection policy.

\footnotetext{
1 Suggested citation: Wu, S. and Wang, W. (2011) Optimal Inspection Policy for Three-state Systems Monitored by Control Charts. Applied Mathematics and Computation, 217 (23). pp. 9810-9819.

Corresponding author. E-mail: shaomin.wu@cranfield.ac.uk
} 


\section{Introduction}

The delay time concept introduced by Christer and Waller [1] regards the failure process as a two-stage process with the first stage (or called normal working state) being from new to the point of a detectable defect arising and the second stage (or called failure delay time state) from this point onward to the failure if no other maintenance interventions were taken. The concept of failure delay time can be used in planning condition-based maintenance policy (see [2], for example): if one can successfully detect the changing point, at which the process shifts from the first stage to the second stage, then we can conduct maintenance during the second stage (before the system fails).

Control charts, a tool from statistical process control (SPC), can be used to detect the changing point. If we use SPC terminologies, the normal working state is said an in-control state and the failure delay time state is said an out-of-control state. Control charts are commonly employed for the purpose of signalling the occurrence of assignable causes every time when the process parameter has shifted. As control charts may produce false signals that incorrectly indicate the state of the system, optimally designing the parameters of control charts to minimizing the cost incurred by the false signals is an important research topic. To this end, various scenarios have been considered by researchers. Tagaras and Lee [3] and Tagaras [4] separated the $\bar{X}$-chart into several zones and optimized the chart for monitoring a process whose deterioration can be classified into two states in which one state requires minor repair and the other requires major repair, and considered economic design of control charts. Cheng et al [5] used the $p$-chart to derive thresholds for aviation inspection. Cassady et al [6] considered economic design of control charts for optimization of preventive maintenance policies for systems. Chan and Wu [7] apply the concept of cumulative count of conforming chart (CCC chart) in inspection and maintenance planning for systems where minor inspection, major inspection, minor maintenance and major maintenance are available. Control charts have also been applied to condition-based maintenance for monitoring two-stage processes that was mentioned in the preceding paragraph (see [8] for example). Other examples of research in this area can also been seen in $[9,10]$. Typical application areas of control charts can be found in continuous manufacturing processes such as canning lines, high precision component machining centres, and electronic item assembly lines.

Commonly, the decision variables in designing control charts include the sampling interval between consecutive sampling points, sample sizes or control limits. The parameters in control charts can be fixed or variable, then we have two different control charts: static and adaptive. A static control chart has fixed parameters, including sample size $n$, sampling interval $h$, lower control limit (LCL) and upper control limit (UCL), whereas an adaptive control chart has at least one of its parameters ( $n, h, \mathrm{LCL}$ and UCL) allowed to be changed based on the information about the state of the process. Compared with the static control charts, the adaptive control chart can utilise the inspection capacity more effectively for a better process control $([11,12,13,14])$. Recent research on adaptive control charts can be found in $[15,16,17,18]$. 
However, in existing literature, there has been little work in investigating the potential of the adaptive control charts to monitor processes where a failure delay time exists. This paper presents the formulations of the expected long-run cost per time unit for such a process monitored by adaptive control charts with variable sampling intervals.

A system with a failure delay time is essentially a three-state system that is a typical case of a multistate system. Research in multistate systems is another interesting topic in reliability theory and engineering, and the reader is referred to $[19,20]$ for more information in this area.

The novelty of this paper lies in the formulations and analysis of the expected cost per time unit for the scenarios where adaptive control charts are applied to monitor three-state systems.

This paper is structured as follows. Section 2 presents assumptions and notation that are used in the paper. In order to compare the efficiency of static and adaptive control charts, Section 3 formulates the expected long-run cost per unit for a system monitored by static and adaptive charts respectively. Section 4 discusses the relationship between the formulas of the expected long-run cost per unit of the static and adaptive charts. Section 5 gives numerical examples to study the sensitive analysis for various parameter settings. Section 6 concludes the findings of this paper.

\section{General assumptions and notation}

This section first presents the usage of the static and adaptive control charts, then makes assumptions for these two charts.

Consider a system with three states: in-control, out-of-control and failed. It produces outputs that can be monitored by a control chart: either a static or an adaptive chart.

Static chart The sampling interval $h$ is fixed. $n$ samples are taken at every $h$ time units. Figure 1 shows a typical example of a control chart with two zones: $Z_{f 0}$ is a central zone, $Z_{f 1}$ is an action zone, and they are defined as: $Z_{f 0}=\{0, \mathrm{UCL}\}, Z_{f 1}=\{\mathrm{UCL}, \infty\}$, for example. The following policy is adopted.

- If the characteristics of the $n$ samples falls in $Z_{f 0}$, no further action will be taken and the next sampling interval will be $h$.

- If the characteristics of the $n$ samples falls in $Z_{f 1}$, then an inspection will be carried out to check the occurrence of assignable causes. If the occurrence is confirmed by the inspection, then a minor repair is performed. Otherwise no further action will be taken and the next sampling interval will be $h$.

Adaptive chart The first sampling interval is $h_{0}$ time units and the samples are taken immediately after the start of the system. After that, $n$ samples are taken at every $h_{0}$ time units. Figure 2 shows a typical example of a control chart with three zones: $Z_{a 0}$ 
is a central zone, $Z_{a 1}$ is a warning zone, $Z_{a 2}$ is an action zone, and they are defined as: $Z_{a 0}=\{0, w\}, Z_{a 1}=\{w, \mathrm{UCL}\}$, and $Z_{a 2}=\{\mathrm{UCL}, \infty\}$, for example. The following policy is adopted.

- If the characteristics of the $n$ samples falls in $Z_{a 0}$, then the next sampling interval will be $h_{0}$ time units;

- If the characteristics of the $n$ samples falls in $Z_{a 1}$, then the next sampling interval will be $h_{1}$ time units, and an inspection will be carried out to check whether the system is in control or not. If the system is confirmed to be in the out-of-control state, then a minor repair is performed to restore the system, otherwise no further action will be taken and the next sampling interval will be $h_{0}$.

- If the characteristics of the $n$ samples falls in $Z_{a 2}$, then an inspection will be carried out to check the occurrence of the assignable causes. If occurrence is confirmed, then a minor repair is performed. Otherwise no further action will be taken and the next sampling interval will be $h_{0}$, which is the same as normal.

The following assumptions are also held for both the static and the adaptive control charts.

(1) Suppose that the system can shift either from an in-control state to an out-of-control state and then to a failure state; or directly from an in-control state to an failure state without going through an out-of-control state. The system itself in a failure state or an out-of-control state cannot restore back to the in-control state without any repair.

(2) When the system is in an in-control state or an out-of-control state, the defective probability of the product is $p_{0}$ or $p_{1}$, respectively, where $p_{1}>p_{0}$.

(3) The inspection is assumed to be perfect in that it can reveal whether the system is in control or not. During the inspection, the system carries on running. As long as the system has been confirmed to be in an out-of-control state, repairmen will carry out a minor repair which can bring the system back to an in-control state. The system is as good as new immediately after the minor repair has been completed, and it is still operating while it is under a minor repair. Once the system fails, repairmen will conduct a major repair that can bring it back to the as-good-as-new state.

(4) For simplicity, times spent on an inspection, minor or major repair are small compared with the sampling interval, and therefore are negligible, but their costs are considered.

Th following notation is also used.

$X_{1}$ : random time from the beginning of an in-control state to the occurrence of an assignable cause;

$f_{1}\left(x_{1}\right)$ : pdf. of $X_{1}$, and $F_{1}\left(x_{1}\right)=\operatorname{Pr}\left(X_{1}<x_{1}\right)$ is the cdf. of $X_{1}$;

$X_{2}$ : random time from the beginning of the out-control state to failure;

$f_{2}\left(x_{2}\right)$ : pdf. of $X_{2}$, and $F_{2}\left(x_{2}\right)=\operatorname{Pr}\left(X_{2}<x_{2}\right)$, cdf. of $X_{2}$;

$n$ : sample size;

$h$ : sampling interval when a static control chart is used; 
$h_{0}$ : a longer sampling interval when an adaptive control chart is used;

$h_{1}$ : a shorter sampling interval when an adaptive control chart is used;

$\alpha_{i}$ : the probability that the characteristics of the $n$ samples falls in $Z_{f 0}$ when the system is in the in-control state for $i=0$, or in the out-of-control state for $i=1$. It is for a static control chart, where $\alpha_{0}+\alpha_{1}=1$.

$\beta_{i j}$ : the probability that the characteristics of the $n$ samples falls in $Z_{a j}(j=0,1,2)$ when the system is in the in-control state for $i=0$, or in the out-of-control state for $i=1$. It is for the adaptive control chart, where $\beta_{00}+\beta_{01}+\beta_{02}=1$ and $\beta_{10}+\beta_{11}+\beta_{12}=1$.

$T_{n}$ : time to the first minor repair with an assignable cause when no control chart is used; $T_{f 1}$ : the random time from the beginning of the in-control state to the occurrence of an assignable cause when a static control chart is used;

$T_{f 2}$ : time between the occurrence of an assignable cause and failure when a static control chart is used;

$T_{a 1}$ : expected time between the start of the system and a minor repair triggered by an inspection due to a warning signal in zone $Z_{a 1}$;

$T_{a 2}$ : expected time between the start of the system and a minor repair triggered by an inspection due to a warning signal in zone $Z_{a 2}$;

$T_{a 3}$ : expected time between the start of the system and a major repair;

$c_{s}$ : sampling cost per item;

$c_{i}$ : inspection cost for a possible assignable cause;

$c_{r 1}$ : cost for a minor repair;

$c_{r 2}$ : cost for a major repair;

$C_{n}$ : time to failure when no control chart is used;

$C_{f 1}, C_{f 2}$ : costs incurred within times $T_{f 1}$ and $T_{f 1}$, respectively;

$C_{a 1}, C_{a 2}, C_{a 3}$ : costs incurred within times $T_{a 1}, T_{a 2}$ and $T_{a 3}$, respectively. They are for an adaptive chart.

Throughout the modelling development, we use the renewal reward theorem, which simply states that the expected long-run cost per time unit is the ratio between the expected renewal cycle cost and expected renewal cycle length [21].

\section{$3 \quad$ Expected long-run cost per time unit}

This section derives the expected long-run cost per time unit for both the static control chart scenario and the adaptive control chart scenario. 


\subsection{No control chart scenario}

In the case there is no control chart used to monitor the system, the expected long-run time is given by

$$
E\left(T_{n}\right)=\int_{0}^{\infty} \int_{0}^{x} x f_{1}\left(x_{1}\right) f_{2}\left(x-x_{1}\right) d x_{1} d x
$$

and the expected cost is

$$
E\left(C_{n}\right)=c_{r 2} \int_{0}^{\infty} \int_{0}^{x} f_{1}\left(x_{1}\right) f_{2}\left(x-x_{1}\right) d x_{1} d x .
$$

The expected long-run cost per time unit is given by

$$
E_{n}(T, C)=\frac{E\left(C_{n}\right)}{E\left(T_{n}\right)}
$$

\subsection{Static control chart scenario}

In the case when a static control chart is employed to monitor the system, the expected time length and expected cost are derived as follows.

\subsubsection{Expected time length}

There can be two renewals: a renewal after a minor repair is conducted, and a renewal after a major repair is completed. The expected renewal cycle length is $E\left(T_{f}\right)=E\left(T_{f 1}\right)+$ $E\left(T_{f 2}\right)$.

Then we have

$$
E\left(T_{f 1}\right)=\sum_{i=1}^{\infty} i h \sum_{j=1}^{i} \alpha_{1}^{i-j}\left(1-\alpha_{1}\right) \int_{(j-1) h}^{j h} f_{1}\left(x_{1}\right)\left[1-F_{2}\left(i h-x_{1}\right)\right] d x_{1} .
$$

Proof. In this case, a renewal occurs after a minor repair is conducted at the $i$ time point. There are $i-j$ false signals followed by one true signal. The false signals wrongly indicate that the system is in an in-control state whereas it is actually in an out-of-control state. The time length is $i h$ that includes $i$ sampling intervals in both the in-control state and the out-of-control state.

The expected value of $T_{f 2}$ is given by

$$
E\left(T_{f 2}\right)=\sum_{i=1}^{\infty} \sum_{j=1}^{i} \alpha_{1}^{i-j} \int_{(i-1) h}^{i h} \int_{(j-1) h}^{\tau_{i j}} x f_{1}\left(x_{1}\right) f_{2}\left(x-x_{1}\right) d x_{1} d x
$$


where $\tau_{i j}=\left\{\begin{array}{l}j h \text { if } j<i \\ x \text { if } j=i\end{array}\right.$

Proof. In this scenario, There are $i-j$ false signals followed by a failure.

The expected renewal cycle length $E\left(T_{f}\right)$ is the summation of $E\left(T_{f 1}\right)$ and $E\left(T_{f 2}\right)$, and it is given by

$$
\begin{aligned}
E\left(T_{f}\right) & =\sum_{i=1}^{\infty} \sum_{j=1}^{i} i h \alpha_{1}^{i-j}\left(1-\alpha_{1}\right) \int_{(j-1) h}^{j h} f_{1}\left(x_{1}\right)\left[1-F_{2}\left(i h-x_{1}\right)\right] d x_{1} \\
& +\sum_{i=1}^{\infty} \sum_{j=1}^{i} \alpha_{1}^{i-j} \int_{(i-1) h}^{i h} \int_{(j-1) h}^{\tau_{i j}} x f_{1}\left(x_{1}\right) f_{2}\left(x-x_{1}\right) d x_{1} d x
\end{aligned}
$$

\subsubsection{Expected renewal cycle cost}

Denote $c_{i j}=(i-1) n c_{s}+\left(1-\alpha_{0}\right)(j-1) c_{i}$ which is the expected cost of samplings and false alarm inspections before a renewal at ih or $x, x \in((i-1) h, i h]$, and the assignable cause occurs at $x_{1}, x_{1} \in((j-1) h, j h]$, then summing over all possible sampling intervals and events, we have

$$
\begin{aligned}
& E\left(C_{f}\right) \\
= & \sum_{i=1}^{\infty} \sum_{j=1}^{i} \alpha_{1}^{i-j}\left(1-\alpha_{1}\right) \int_{(j-1) h}^{j h}\left(c_{i j}+n c_{s}+c_{i}+c_{r 1}\right) f_{1}\left(x_{1}\right)\left[1-F_{2}\left(i h-x_{1}\right)\right] d x_{1} \\
+ & \sum_{i=1}^{\infty} \sum_{j=1}^{i} \alpha_{1}^{i-j} \int_{(i-1) h}^{i h} \int_{(j-1) h}^{\tau_{i j}}\left(c_{i j}+c_{r 2}\right) f_{1}\left(x_{1}\right) f_{2}\left(x-x_{1}\right) d x_{1} d x .
\end{aligned}
$$

Hence, the expected long-run cost per time unit is given by

$$
E_{f}(T, C)=\frac{E\left(C_{f}\right)}{E\left(T_{f}\right)}
$$

\subsection{Adaptive control chart scenario}

There are two possible kinds of transition of the system: a transition from the in-control state to the out-of-control state detected by signals either in the warning zone or in the action zone, and a transition from the out-of-control state to failure. We assume that any level of maintenance can renew the system. 


\subsubsection{Expected renewal cycle length}

The expected renewal cycle length is $E\left(T_{a}\right)=E\left(T_{a 1}\right)+E\left(T_{a 2}\right)+E\left(T_{a 3}\right)$, which is explained as follows.

The expected time between the start and a minor repair triggered by an inspection due to a signal in zone $Z_{a 1}$ is given by

$$
\begin{aligned}
& E\left(T_{a 1}\right)=\sum_{k_{1}=1}^{\infty} \sum_{k_{2}=1}^{k_{1}} \sum_{k_{3}=0}^{k_{1}-k_{2}}\left\{\beta_{10}^{k_{1}-k_{2}} \beta_{11}^{k_{3}} \beta_{11}\left(1-\beta_{10}\right) H_{0} \int_{H_{1}-h_{0}-h_{1}}^{H_{1}-h_{1}} f_{1}\left(x_{1}\right)\left(1-F_{2}\left(H_{0}-x_{1}\right)\right) d x_{1}\right. \\
+ & \left.\beta_{01} \beta_{10}^{k_{1}-k_{2}+1} \beta_{11}^{k_{3}+1}\left(1-\beta_{10}\right)\left(H_{0}+h_{0}+h_{1}\right) \int_{H_{1}-h_{1}}^{H_{1}} f_{1}\left(x_{1}\right)\left(1-F_{2}\left(H_{0}+h_{0}+h_{1}-x_{1}\right)\right) d x_{1}\right\} \\
+ & \sum_{k_{2}=0}^{\infty}\left\{\beta_{01}\left(1-\beta_{10}\right) H_{2} \int_{H_{2}-h_{1}}^{H_{2}} f_{1}\left(x_{1}\right)\left(1-F_{2}\left(H_{2}-x_{1}\right)\right) d x_{1}\right\},
\end{aligned}
$$

where $H_{0}=k_{1} h_{0}+\left(\beta_{01}\left(k_{2}-1\right)+k_{3}+1\right) h_{1}, H_{1}=k_{2} h_{0}+\left(\beta_{01}\left(k_{2}-1\right)+1\right) h_{1}$, and $H_{2}=\left(k_{2}+1\right) h_{0}+\left(\beta_{01} k_{2}+1\right) h_{1}$.

Proof. The description of three units in Eq (9) is given below. There are three scenarios for the system transiting from an in-control state to an out-of-control state. These three states correspond to the following the three units in Eq (9).

Denote $k_{1}$ as the total number of longer sampling intervals (ie. $h_{0}$ ) in both the in-control and out-of-control states, $k_{2}$ as the total number of longer sampling intervals in an incontrol state, and $k_{3}$ as the number of false signals followed by true ones in the out-ofcontrol state.

Unit 1 The system transits from the in-control state to the out-of-control state in a longer sampling interval $h_{0}$. Apparently, the effectiveness of the control chart is associated with the length of an out-of-control state, but independent of the length of the in-control state.

When the system is in the out-of-control state, there might be false signals (with a probability of $\beta_{10}^{k_{1}-k_{2}-k_{3}}$ ) in $h_{0}$, or true signals in $h_{0}$ but followed by false signals in $h_{1}$ (with a probability of $\left(\beta_{11} \beta_{10}\right)^{k_{3}}$ ). These two scenarios make up an event with a probability of $\left(\beta_{10}\right)^{k_{1}-k_{2}-k_{3}}\left(\beta_{11} \beta_{10}\right)^{k_{3}}$ (or $\left.\beta_{10}^{k_{1}-k_{2}} \beta_{11}^{k_{3}}\right)$, and take time $\left(k_{1}-k_{2}\right) h_{0}+k_{3} h_{1}$. Eventually, a correct signal in $h_{0}$ is followed by another correct signal in $h_{1}$, which has a probability of $\beta_{11}\left(1-\beta_{10}\right)$ and a time length of $h_{0}+h_{1}$.

Before the system has transited from the in-control state to the out-of-control state, the time length is $\left(k_{2}-1\right) h_{0}+\beta_{01}\left(k_{2}-1\right) h_{1}$. Hence, the total length is $k_{1} h_{0}+\left(\beta_{01}\left(k_{2}-\right.\right.$ $\left.1)+k_{3}+1\right) h_{1}=H_{0}$. The transition occurs in the time interval $\left(\left(k_{2}-1\right) h_{0}+\beta_{01}\left(k_{2}-\right.\right.$ 1) $\left.h_{1}, k_{2} h_{0}+\beta_{01}\left(k_{2}-1\right) h_{1}\right)$, or $\left(H_{1}-h_{0}-h_{1}, H_{1}-h_{1}\right)$.

Unit 2 The system might also transit from the in-control state to the out-of-control state within a short sampling interval $h_{1}$ after a false signal appears in the in-control state, but a correct signal follows. This event has a probability of $\beta_{01} \beta_{10}$. A correct signal can 
appear in the sample of this short sampling interval. This event has a probability of $\beta_{01}\left(1-\beta_{10}\right)$ and a time length of $h_{0}+h_{1}$. The time length of the system in the in-control state is $\left(k_{2}-1\right) h_{0}+\beta_{01}\left(k_{2}-1\right) h_{1}$, then the transition from the in-control state to the out-of-control state occurs in $\left(H_{1}-h_{1}, H_{1}\right)$ (where $\left.H_{1}=k_{2} h_{0}+\beta_{01}\left(k_{2}-1\right) h_{1}+h_{1}\right)$. After the system has transited to the out-of-control state, the probability of the appearance of a correct signal is given by $\beta_{10}^{k_{1}-k_{2}-k_{3}}\left(\beta_{11} \beta_{10}\right)^{k_{3}} \beta_{11}\left(1-\beta_{10}\right)\left(\right.$ or $\left.\beta_{10}^{k_{1}-k_{2}} \beta_{11}^{k_{3}+1}\left(1-\beta_{10}\right)\right)$ and has a time length of $H_{0}+h_{0}+h_{1}$.

Unit 3 When the system is in the in-control state, a false signal appears in a longer sampling interval $h_{0}$. Then a short sampling interval is followed and the system transits to the out-of-control state in this short sampling interval, and then a true signal appears. This event has a probability of $\beta_{01}\left(1-\beta_{10}\right)$.

The expected time between the start and a minor repair triggered by an inspection due to a signal in zone $Z_{a 2}$ is given by

$$
E\left(T_{a 2}\right)=\sum_{k_{1}=1}^{\infty} \sum_{k_{2}=1}^{k_{1}} \sum_{k_{3}=0}^{k_{1}-k_{2}}\left\{\beta_{10}^{k_{1}-k_{2}} \beta_{11}^{k_{3}} \beta_{12}\left(H_{0}-h_{1}\right) \int_{H_{1}-h_{0}-h_{1}}^{H_{1}-h_{1}} f_{1}\left(x_{1}\right)\left(1-F_{2}\left(H_{0}-h_{1}-x_{1}\right)\right) d x_{1}\right\} .
$$

Proof. The proof is similar to that of $E\left(T_{a 1}\right)$, apart from the appearance of the out-ofcontrol signals in a longer interval $h_{0}$ in this case.

The expected time between the start and a major repair is given by

$$
\begin{aligned}
& E\left(T_{a 3}\right)=\sum_{k_{1}=1}^{\infty} \sum_{k_{2}=1}^{k_{1}} \sum_{k_{3}=0}^{k_{1}-k_{2}} \beta_{10}^{k_{1}-k_{2}} \beta_{11}^{k_{3}}\left\{\int_{H_{0}-h_{0}-h_{1}}^{H_{0}-h_{1}} \int_{H_{1}-h_{0}-h_{1}}^{\tau_{k_{1} k_{2} k_{3}}} x f_{1}\left(x_{1}\right) f_{2}\left(x-x_{1}\right) d x_{1} d x\right. \\
& +\beta_{11} \int_{H_{0}-h_{1}}^{H_{0}} \int_{H_{1}-h_{0}-h_{1}}^{\tau_{k_{1} k_{2} k_{3}}} x f_{1}\left(x_{1}\right) f_{2}\left(x-x_{1}\right) d x_{1} d x \\
& +\int_{H_{0}-h_{0}-h_{1}}^{H_{0}-h_{1}} \int_{H_{1}-h_{1}}^{\tau_{k_{1} k_{2} k_{3}}^{\prime}} x f_{1}\left(x_{1}\right) f_{2}\left(x-x_{1}\right) d x_{1} d x \\
& \left.+\beta_{11} \int_{H_{0}-h_{1}}^{H_{0}} \int_{H_{1}-h_{1}}^{\tau_{\tau_{1} k_{2} k_{3}}^{\prime}} x f_{1}\left(x_{1}\right) f_{2}\left(x-x_{1}\right) d x_{1} d x\right\} . \\
& \text { where } \tau_{k_{1} k_{2} k_{3}}=\left\{\begin{array}{l}
H_{1}-h_{1} \text { if } k_{1}-k_{2} \neq 0 \\
x \quad \text { if } k_{1}-k_{2}=0,
\end{array} \text { and } \tau_{k_{1} k_{2} k_{3}}^{\prime}= \begin{cases}H_{1} \text { if } k_{1}-k_{2} \neq 0 \\
x & \text { if } k_{1}-k_{2}=0 .\end{cases} \right.
\end{aligned}
$$

Proof. The system might transit from the in-control state to the out-of-control state either in a longer sampling interval $h_{0}$ or in a shorter sampling interval $h_{1}$, and the system can then fail in either $h_{0}$ or $h_{1}$ as well, which creates four scenarios. The first two components in Eq (11) correspond to the scenarios when the transition from the in-control state to the out-of-control state occurs in a $h_{0}$, and the last two components in Eq (11) correspond to the scenarios when the transition from the in-control state to the out-of-control state occurs in a $h_{1}$. 
The first component in $\mathrm{Eq}(11)$ is the scenario when the two transitions (i.e., from the incontrol state to the out-of-control state and then fail) occur in longer sampling intervals. The second component means that a correct signal appears in a longer sampling interval $h_{0}$ (with a probability $\beta_{11}$ ) followed by a shorter sampling interval $h_{1}$ for confirmation, but the system fails within this $h_{1}$. The third component means that the transition from the in-control state to the out-of-control state occurs (with a probability $\beta_{01}$ followed by a shorter sampling interval). The last component means that the two scenarios occur in short sampling intervals.

\subsubsection{Expected renewal cycle cost}

The costs incurred during periods $E\left(T_{f 1}\right), E\left(T_{f 2}\right)$, and $E\left(T_{f 3}\right)$ are derived in the following.

$$
\begin{aligned}
E\left(C_{a 1}\right) & =\sum_{k_{1}=1}^{\infty} \sum_{k_{2}=1}^{k_{1}} \sum_{k_{3}=0}^{k_{1}-k_{2}}\left\{\beta_{10}^{k_{1}-k_{2}} \beta_{11}^{k_{3}} \beta_{11}\left(1-\beta_{10}\right) \int_{H_{1}-h_{0}-h_{1}}^{H_{1}-h_{1}} C_{0} f_{1}\left(x_{1}\right)\left(1-F_{2}\left(H_{0}-x_{1}\right)\right) d x_{1}\right. \\
& \left.+\beta_{01} \beta_{10} \beta_{10}^{k_{1}-k_{2}} \beta_{11}^{k_{3}} \beta_{11}\left(1-\beta_{10}\right) \int_{H_{1}-h_{1}}^{H_{1}} C_{1} f_{1}\left(x_{1}\right)\left(1-F_{2}\left(H_{0}+h_{0}+h_{1}-x_{1}\right)\right) d x_{1}\right\} \\
& +\sum_{k_{2}=0}^{\infty}\left\{\beta_{01}\left(1-\beta_{10}\right) \int_{H_{2}-h_{1}}^{H_{2}} C_{2} f_{1}\left(x_{1}\right)\left(1-F_{2}\left(H_{2}-x_{1}\right)\right) d x_{1}\right\},
\end{aligned}
$$

where $C_{0}=\left(k_{1}+\beta_{01}\left(k_{2}-1\right)+k_{3}+1\right) n c_{s}+\left(\left(\beta_{02}+\beta_{01}\left(1-\beta_{00}\right)\right)\left(k_{2}-1\right)+1\right) c_{i}+c_{r 1}$, $C_{1}=C_{0}+2 n c_{s}$, and $C_{2}=k_{2} n c_{s}+\beta_{01} k_{2} n c_{s}+2 n c_{s}+\left(\beta_{02}+\beta_{01}\left(1-\beta_{00}\right)\right) k_{2} c_{i}+c_{i}+c_{r 1}$.

Proof. After the system transited from an in-control state to an out-of-control state within a longer interval $h_{0}$, there will be two possible scenarios before two warning signals appear consecutively in a longer interval and shorter interval, respectively. The first scenario is incorrect signals (with a probability of $\beta_{10}$ ) appears, the second scenario is a correct signal followed by an incorrect signal (with a probability of $\beta_{11} \beta_{10}$ ). These two scenarios makes up an event with a probability of $\left(\beta_{10}\right)^{k_{1}-k_{2}-k_{3}}\left(\beta_{11} \beta_{10}\right)^{k_{3}} \beta_{11}\left(1-\beta_{10}\right)$, and the event incurs sampling cost $\left(k_{1}-k_{2}+1\right) n c_{s}+\left(k_{3}+1\right) n c_{s}$. Before the system has transited from the incontrol state to the out-of-control state, the sampling cost is $k_{2} n c_{s}+\beta_{01} k_{2} n c_{s}$, inspection $\operatorname{cost}\left(\beta_{02}+\beta_{01}\left(1-\beta_{00}\right) k_{2}+1\right) c_{i}$ and level 1 repair cost $c_{r 1}$. Hence, the sub-total cost is $C_{0}$.

The system might also transit from the in-control state to the out-of-control state within a short interval $h_{1}$ after a false signal appear in the in-control state. This event incurs cost $n c_{s}+c_{i}+c_{r 1}$. The cost incurred before the transition is $k_{2} n c_{s}+\beta_{01} k_{2} n c_{s}+\beta_{01} \beta_{01} k_{2} c_{i}$. The sub-total cost is $C_{1}$.

A similar explanation to the third component in $\mathrm{Eq}(12)$ can be given. 
Similarly, we have

$$
E\left(C_{a 2}\right)=\sum_{k_{1}=1}^{\infty} \sum_{k_{2}=1}^{k_{1}} \sum_{k_{3}=0}^{k_{1}-k_{2}}\left\{\beta_{10}^{k_{1}-k_{2}} \beta_{11}^{k_{3}} \beta_{12} \int_{H_{1}-h_{0}-h_{1}}^{H_{1}-h_{1}} C_{3} f_{1}\left(x_{1}\right)\left(1-F_{2}\left(H_{0}-h_{0}-x_{1}\right)\right) d x_{1}\right\}
$$

where $C_{3}=C_{0}-n c_{s}$. Finally, we have

$$
\begin{aligned}
E\left(C_{a 3}\right) & =\sum_{k_{1}=1}^{\infty} \sum_{k_{2}=1}^{k_{1}} \sum_{k_{3}=0}^{k_{1}-k_{2}} \beta_{10}^{k_{1}-k_{2}} \beta_{11}^{k_{3}}\left\{\int_{H_{0}-h_{0}-h_{1}}^{H_{0}-h_{1}} \int_{H_{1}-h_{0}-h_{1}}^{\tau_{k_{1} k_{2} k_{3}}} C_{4} f_{1}\left(x_{1}\right) f_{2}\left(x-x_{1}\right) d x_{1} d x\right. \\
& +\beta_{11} \int_{H_{0}-h_{1}}^{H_{0}} \int_{H_{1}-h_{0}-h_{1}}^{\tau_{k_{1} k_{2} k_{3}}} C_{5} f_{1}\left(x_{1}\right) f_{2}\left(x-x_{1}\right) d x_{1} d x \\
& +\int_{H_{0}-h_{0}-h_{1}}^{H_{0}-h_{1}} \int_{H_{1}-h_{1}}^{\tau_{k_{1} k_{2} k_{3}}^{\prime}} C_{6} f_{1}\left(x_{1}\right) f_{2}\left(x-x_{1}\right) d x_{1} d x \\
& \left.+\beta_{11} \int_{H_{0}-h_{1}}^{H_{0}} \int_{H_{1}-h_{1}}^{\tau_{k_{1} k_{2} k_{3}}^{\prime}} C_{7} f_{1}\left(x_{1}\right) f_{2}\left(x-x_{1}\right) d x_{1} d x\right\} .
\end{aligned}
$$

where $C_{4}=\left(k_{1}+\beta_{01}\left(k_{2}-1\right)+k_{3}-1\right) n c_{s}+\left(\left(\beta_{02}+\beta_{01}\left(1-\beta_{00}\right)\right)\left(k_{2}-1\right)\right) c_{i}+c_{r 2}, C_{5}=$ $C_{4}+n c_{s}+c_{i}, C_{6}=C_{4}$, and $C_{7}=C_{5}$.

Hence, the expected cost per time unit is given by

$$
E_{a}(T, C)=\frac{E\left(C_{a 1}\right)+E\left(C_{a 2}\right)+E\left(C_{a 3}\right)}{E\left(T_{a 1}\right)+E\left(T_{a 2}\right)+E\left(T_{a 3}\right)}
$$

\section{Discussion}

\subsection{Comparison between the two types of control charts}

For the static control chart, if we set $\mathrm{UCL}=\infty$ and $n=0$, then Eq. (6) becomes

$$
\begin{aligned}
E\left(T_{f}\right) & =\sum_{i=1}^{\infty} \sum_{j=1}^{i} \int_{(i-1) h}^{i h} \int_{(j-1) h}^{\tau_{i j}} x f_{1}\left(x_{1}\right) f_{2}\left(x-x_{1}\right) d x_{1} d x \\
& =\int_{0}^{\infty} x \int_{0}^{x} f_{1}\left(x_{1}\right) f_{2}\left(x-x_{1}\right) d x_{1} d x
\end{aligned}
$$

Eq. (7) becomes 


$$
\begin{aligned}
E\left(C_{f}\right) & =\sum_{i=1}^{\infty} \sum_{j=1}^{i} \int_{(i-1) h}^{i h} \int_{(j-1) h}^{\tau_{i j}} c_{r 2} f_{1}\left(x_{1}\right) f_{2}\left(x-x_{1}\right) d x_{1} d x \\
& =c_{r 2} \int_{0}^{\infty} \int_{0}^{x} f_{1}\left(x_{1}\right) f_{2}\left(x-x_{1}\right) d x_{1} d x
\end{aligned}
$$

Then the expected cost per time unit, $\frac{E\left(C_{f}\right)}{E\left(T_{f}\right)}$ based on Eq (17) and Eq (16), is the same as $\mathrm{Eq}(3)$ where no control chart is employed.

Similarly, if we set $Z_{a 1}=\phi$, or $\beta_{01}=\beta_{11}=0$, then the adaptive np chart becomes the static np control chart. Then we have $E\left(T_{a 1}\right)=E\left(C_{a 1}\right)=0, E\left(T_{a 2}\right)=E\left(T_{f 1}\right)$, $E\left(T_{a 3}\right)=E\left(T_{f 2}\right)$, and $E\left(C_{a 2}\right)+E\left(C_{a 3}\right)=E\left(C_{f}\right)$. The expected costs per time unit from the adaptive and static np control charts are the same.

By minimising Eq. (8) and Eq. (15), we can obtain the optimal $h$ when using static control charts and $h_{0}$ and $h_{1}$ when using adaptive control charts, respectively. For given cdf's $\left(F_{1}\left(x_{1}\right)\right.$ and $\left.F_{2}\left(x_{2}\right)\right)$ and costs, $h, h_{0}$ and $h_{1}$ can be obtained by using heuristic search algorithms such as genetic algorithms and simulated annealing [19].

\subsection{Design procedure}

When designing an optimal inspection policy, one can adopt the following steps.

It is reasonable to assume that in practice we can obtain cost parameters $c_{s}, c_{i}, c_{r 1}, c_{r 2}$, cumulative distribution functions $F_{1}\left(x_{1}\right)$ and $F_{2}\left(x_{2}\right)$.

If the values of $\alpha_{0}, \alpha_{0}$ (where $\left.\alpha_{0}+\alpha_{1}=1\right), \beta_{00}, \beta_{01}$, and $\beta_{02}$ (where $\beta_{00}+\beta_{01}+\beta_{02}=1$ ), $\beta_{10}, \beta_{11}$, and $\beta_{12}$ (where $\beta_{10}+\beta_{11}+\beta_{12}=1$ ) are given, we can minimise the expected costs in Eq. (8) and Eq. (15), respectively, to obtain the optimal values $h_{0}, h_{1}$, and $n$.

\section{$5 \quad$ Numerical experiments}

In this section, we use both artificial generated data and real data for validating the approaches proposed in this paper.

\subsection{Artificial generated data}

Assume that $F_{1}\left(x_{1}\right)=1-\exp \left(-\left(\frac{x_{1}}{300}\right)^{2.5}\right)$, and $F_{2}\left(x_{2}\right)=1-\exp \left(-\left(\frac{x_{2}}{200}\right)^{4}\right)$. We also assume the parameter values in Table 1 in this example. 
With the parameter values listed in Table 1, we can compare the values of the expected cost per time unit derived by Eq (8) and Eq (15). Table 2 shows the expected cost per time unit against sampling intervals $h$ for the system monitored with the static control chart. Table 3 shows the expected cost per time unit against longer sampling intervals $h_{0}$ and shorter sampling intervals $h_{1}$. For example, when $h_{0}$ is 80 , then $h_{1}=48$ makes the expected value per time unit minimised (the minimised value is 3.510). This experiment offers the following optimal solutions for the static and adaptive control charts, respectively.

- When $h$ is 88 , the expected cost per time unit achieves the minimal value 3.531847 for the static control chart.

- When $h_{0}=104$, and $h_{1}=9$, the expected long-run cost per time unit is 3.306189 for the adaptive control chart.

It can be seen from the above solutions that the adaptive control chart is superior over the static control chart. However, for given sampling intervals, for example, $h_{0}=h_{1}=h=40$, then the optimal expected cost per time unit, $E_{a}(T, C)$, incurred in the static control chart case can be smaller than in the adaptive control chart case. This is because there is cost added by an additional sampling interval $h_{1}$ immediately followed by sampling interval with another length $h_{0}$ in the adaptive control chart case.

Table 3 also indicates that for minimising the expected long-run cost per time unit a larger value $h_{0}$ should be followed by a smaller value $h_{1}$, whereas a smaller value $h_{0}$ should be followed by a larger value $h_{1}$, although in both cases $h_{0}>h_{1}$. For example, when $h_{0}$ is smaller than 60 , every long sampling interval $h_{0}$ should be followed by the same length of short sampling interval $h_{1}$ : the values of the two columns $h_{1}$ and $h_{0}$ are the same. When $h_{0}$ is larger than 60 , every long sampling interval $h_{0}$ should be followed by the smaller length of short sampling interval $h_{1}$.

\subsection{Vibration data of bearings}

Figure 3 shows the data of the overall vibration level in root mean square (RMS) value of six bearings. The data were collected from an accelerated laboratory experiment. It has been generally accepted in the case of vibration monitoring that a consistent increase in the overall vibration level is a good indicator of the presence of a hidden defect which indicates clearly a two-stage process $[2,8]$.

If we use $\mathrm{RMS}=4.15$ as a threshold: the RMS larger than 4.15 implies that the process is in the failure delay time and the RMS less than 4.15 suggests that the process is in the normal working stage. We can obtain the exact time before each bearing passes over the threshold. By treating these data as time-to-failure data and using maximum likelihood estimation, we can obtain that $F_{1}\left(x_{1}\right)=1-\exp \left(-\left(\frac{x_{1}}{161.58}\right)^{0.72}\right)$. Similarly, we can obtain $F_{2}\left(x_{2}\right)=1-\exp \left(-\left(\frac{x_{2}}{78.62}\right)^{1.80}\right)$. Assume $c_{s}=0.08, c_{i}=80, c_{r 1}=1500, c_{r 2}=5000$, and $n=5$, and the rest parameter values are the same as those in Table 1 . Then we can obtain the optimal solution $E_{a}(T, C)=7.75$ when an adaptive control chart with $h_{0}=13$ and $h_{1}=1$ 
is used, whereas the optimal value $E_{f}(T, C)=7.95$ when a static control with a sample interval $h=9$ is used.

\section{Conclusions}

In this paper, we formulated the expected long-run cost per time unit for a three-state system monitored by an adaptive control chart with variable parameters and a control chart with static parameters. The adaptive control chart has variable sampling intervals: if the control chart signals that the system is near out-of-control at a time point, the subsequent sampling will be conducted in a shorter time period.

The numerical example shows that applying an adaptive control chart to monitor a threestate system is more cost effective than applying a static one. It also shows that for economically maintaining a three state system, an adaptive control chart should be designed to be that the length of the longer sampling interval should be inverse proportional to the length of the shorter sampling interval.

\section{Acknowledgement}

We are grateful to the anonymous referees for their valuable comments and suggestions that result in improvement of the quality of this paper.

\section{References}

[1] Christer, A.H., Waller, W.M., 1984. Reducing production downtime using delay-time analysis. Journal of the Operational Research Society 35 (6), 499-512.

[2] Wang, W., 2002. A model to predict the residual life of rolling element bearings given monitored condition information to date. IMA Journal Management Mathematics 13 (1), $3-16$.

[3] Tagaras, G., Lee, H.L., 1988. Economic design of control charts with different control limits for different assignable causes. Management Science 34(11), 1347-1366.

[4] Tagaras, G., Lee, H.L., 1989. Approximate semi-economic design of control charts with multiple control limits. Naval Research Logistics 36, 337-353.

[5] Cheng, A.Y, Liu, R.Y., Luxhøj, J.T., 1997. Thresholds for safety inspection measurements based on control charts. International Journal of Reliability, Quality and Safety Engineering $4(2), 205-225$. 
[6] Cassady, C.R, Bowen, R.O, Liew, L, Pohl, E.A., 2000. Preventive maintenance and statistical process control: a preliminary inspection, IIE Transactions 32(6), 471-478.

[7] Chan, L.-Y., Wu, S. Optimal design for inspection and maintenance policy based on the CCC chart, Computers \& Industrial Engineering 57(3), 667-676.

[8] Wang, W., Zhang, W., 2010. Early defect identification: Application of statistical process control methods. Journal of Quality in Maintenance Engineering 14(3), 225-236.

[9] Jolayemi, J. K., 2000, An optimal design of multi-attribute control charts for processes subject to a multiplicity of assignable causes. Applied Mathematics and Computation, 114(23), $187-203$.

[10] Issam, B.K. Mohamed, L. Support vector regression based residual MCUSUM control chart for autocorrelated process. Applied Mathematics and Computation, 201(1-2), 565-574.

[11] De Magalhes, M.S., Epprecht, E.K., Costa, A.F.B., 2001. Economic design of a Vp $\bar{X}$ chart. International Journal of Production Economics 74, 191-200.

[12] Chen,Y.K., Hsieh,K.L., Chang,C.C., 2007. Economic design of the VSSI control charts for correlated data. International Journal of Production Economics 107(2), 528-539.

[13] Zhang, S., Wu, Z., 2007. A CUSUM scheme with variable sample sizes for monitoring process shifts. International Journal of Advanced Manufacturing Technology, 33(9-10), 977-987.

[14] Liu, J.Y., Xie, M., Goh, T.N., Liu, Q.H., Yang, Z.H., 2006. Cumulative count of conforming chart with variable sampling intervals. International Journal of Production Economics, 101 (2), pp. 286-297.

[15] Epprecht, E.K., Costa, A.F.B., 2001. Adaptive sample size control charts for attributes. Quality Engineering 13(3), 465-473.

[16] Epprecht, E.K., Costa, A.F.B., Mendes, F.C.T., 2003. Adaptive control charts for attributes. IIE Transactions 35 (6), 567-582.

[17] Wu, Z., Luo, H., 2004. Optimal design of the adaptive sample size and sampling interval np control chart. Quality and Reliability Engineering International 20(6), 553-570.

[18] Luo, Y., Li, Z., Wang, Z. Adaptive CUSUM control chart with variable sampling intervals (2009) Computational Statistics and Data Analysis, 53 (7), pp. 2693-2701

[19] Wu, S., Chan, L.-Y., 2003. Performance utility of multi-state systems. IEEE Transactions On Reliability 52, 14-21.

[20] Wu, S., 2005. Joint importance of multistate systems. Computers and Industrial Engineering 49, 63-75.

[21] Ross, S. M, 2007. Introduction to probability models, Academic Press, London. 


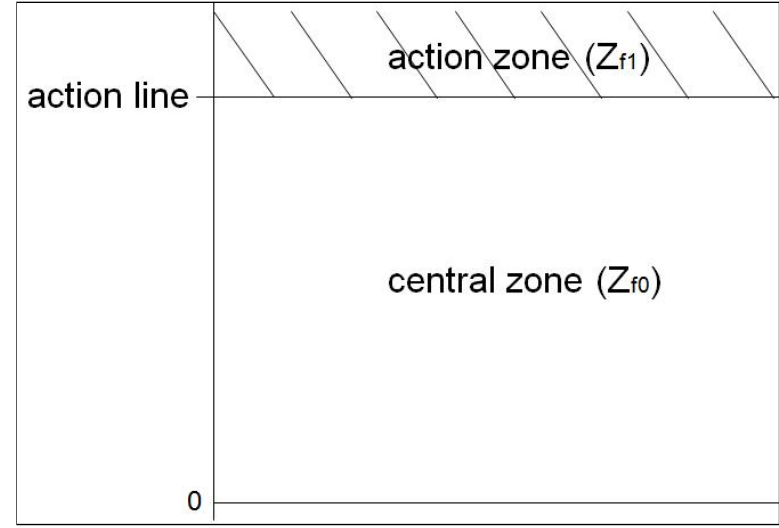

Fig. 1. A control chart with two zones

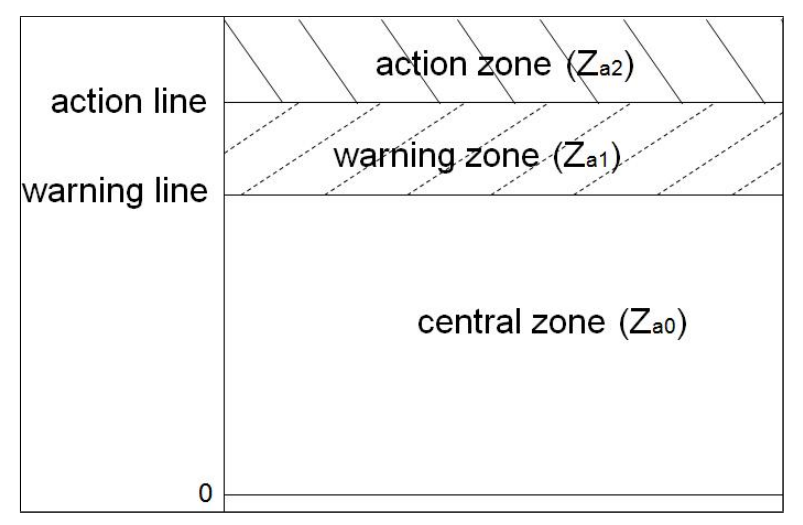

Fig. 2. A control chart with three zones

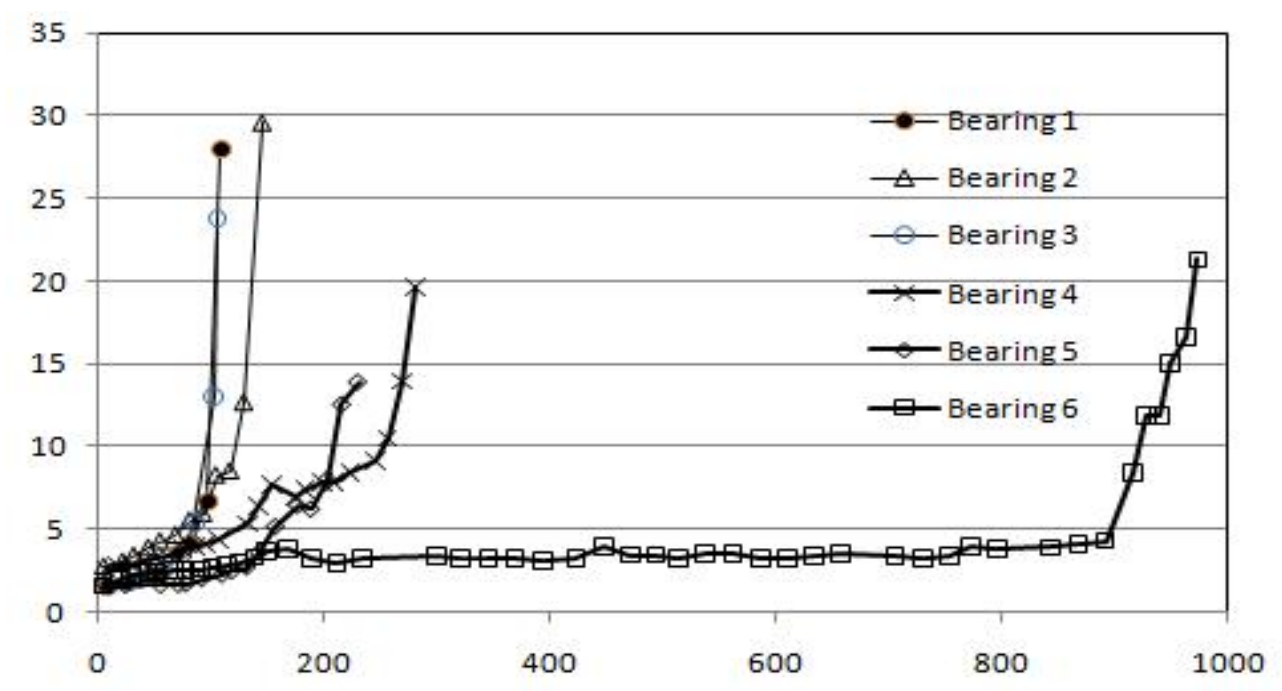

Fig. 3. Vibration data of six bearings. (X-axis: operating hours since new; Y-axis: vibration levels (rms)) 
Table 1

Parameters used in the numerical example.

\begin{tabular}{ccccccccccccc}
\hline$\alpha_{0}$ & $\alpha_{1}$ & $\beta_{00}$ & $\beta_{01}$ & $\beta_{02}$ & $\beta_{10}$ & $\beta_{11}$ & $\beta_{12}$ & $c_{s}$ & $c_{i}$ & $c_{r 1}$ & $c_{r 2}$ & $\mathrm{n}$ \\
\hline 0.95 & 0.05 & 0.833 & 0.147 & 0.02 & 0.02 & 0.08 & 0.9 & 1 & 100 & 500 & 5000 & 100 \\
\hline
\end{tabular}

Table 2

The expected cost per time unit of different sampling intervals.

\begin{tabular}{cc|cc|cc|cc|cc|cc}
\hline$h$ & cost & $h$ & cost & $h$ & cost & $h$ & cost & $h$ & cost & $h$ & cost \\
\hline 40 & 4.659 & 60 & 3.842 & 80 & 3.586 & 100 & 3.613 & 120 & 3.805 & 140 & 4.092 \\
45 & 4.367 & 65 & 3.742 & 85 & 3.572 & 105 & 3.649 & 125 & 3.870 & 145 & 4.174 \\
50 & 4.145 & 70 & 3.668 & 90 & 3.573 & 110 & 3.694 & 130 & 3.940 & 150 & 4.259 \\
55 & 3.974 & 75 & 3.617 & 95 & 3.587 & 115 & 3.746 & 135 & 4.014 & & \\
\hline
\end{tabular}

Table 3

The expected cost per time unit with changing longer and shorter sampling intervals.

\begin{tabular}{ccc|ccc|ccc|ccc}
\hline$h_{0}$ & $h_{1}$ & $E_{a}(T, C)$ & $h_{0}$ & $h_{1}$ & $E_{a}(T, C)$ & $h_{0}$ & $h_{1}$ & $E_{a}(T, C)$ & $h_{0}$ & $h_{1}$ & $E_{a}(T, C)$ \\
\hline 40 & 36 & 4.684 & 70 & 49 & 3.646 & 100 & 10 & 3.374 & 130 & 3 & 3.431 \\
45 & 45 & 4.391 & 75 & 52 & 3.572 & 105 & 9 & 3.354 & 135 & 3 & 3.471 \\
50 & 50 & 4.162 & 80 & 48 & 3.510 & 110 & 4 & 3.351 & 140 & 3 & 3.520 \\
55 & 55 & 3.983 & 85 & 34 & 3.457 & 115 & 4 & 3.357 & 145 & 3 & 3.577 \\
60 & 60 & 3.842 & 90 & 27 & 3.422 & 120 & 4 & 3.372 & 150 & 3 & 3.642 \\
65 & 58 & 3.730 & 95 & 19 & 3.387 & 125 & 3 & 3.398 & & & \\
\hline
\end{tabular}

